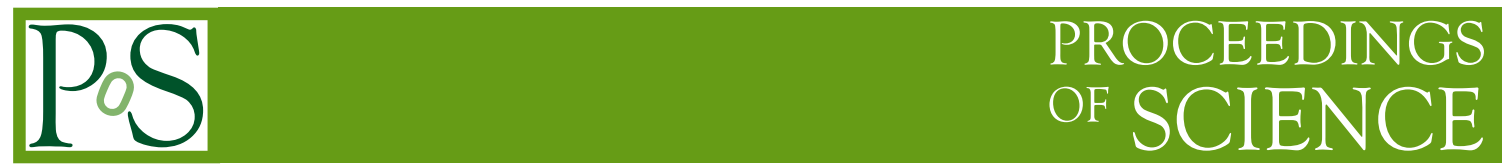

\title{
What can be learned from light meson decays
}

\author{
Christoph Florian Redmer*i \\ Department of Physics and Astronomy, Uppsala University, Sweden \\ E-mail: christoph.redmer@physics.uu.se
}

\begin{abstract}
The physics program investigated by the WASA-at-COSY collaboration focuses on the investigation of light meson decays with the aim of performing precision tests of Chiral Perturbation Theory and to scrutinize symmetries and symmetry breaking mechanisms in hadronic systems using rare decays. Additionally, hadron structure is studied through form factors. Contributions to dark matter searches are made by studying very rare leptonic decay modes of light pseudo scalar mesons.

The light meson decay program at the WASA facility at COSY will be outlined and some highlights of $\pi^{0}, \eta$ decay studies will be presented.
\end{abstract}

8th International Conference on Nuclear Physics at Storage Rings-Stori11,

October 9-14, 2011

Laboratori Nazionali di Frascati dell'INFN, Italy

*Speaker.

${ }^{\dagger}$ on behalf of the WASA-at-COSY Collaboration 


\section{Introduction}

Decays of the light mesons $\pi^{0}, \eta$ and $\omega$ decays are one of the main tools for the WASA-atCOSY collaboration to follow a physics program that focuses on the investigation of symmetries and symmetry breaking patterns [1]. The studies of the light meson decays can be grouped in three topics.

The isospin symmetry breaking decays of $\eta$ into three pions allow to perform precision tests of chiral perturbation theory. Similar tests can be performed with the decays $\eta \rightarrow \pi^{+} \pi^{-} \gamma$ and $\omega \rightarrow \pi^{+} \pi^{-} \pi^{0}$, to probe the anomalous sector of QCD originating from the breaking of chiral symmetry due to quantization. The decay $\eta \rightarrow \pi^{0} \gamma \gamma$ is specially suited to test the higher orders of Chiral Perturbation Theory, since only the terms of the Lagrangian of $\mathscr{O}\left(p^{6}\right)$ contribute to the decay.

A second issue in the studies of light meson decays are the transition form factors. In the single Dalitz decays $\pi^{0}, \eta \rightarrow e^{+} e^{-} \gamma$ and $\omega \rightarrow \pi^{0} e^{+} e^{-}$and the double Dalitz decays $\pi^{0}, \eta \rightarrow e^{+} e^{-} e^{+} e^{-}$ the transition form factors can be determined in the time-like region. They contain information about the electromagnetic structure of the decaying neutral mesons and are important input to the calculations of the $g-2$ of the muon.

The decays of $\pi^{0}, \eta$ and $\omega$ mesons also provide the possibility to tests predictions of the Standard Model and to search for physics beyond it. The decay $\eta \rightarrow \pi^{0} e^{+} e^{-}$provides a direct test for $C$ violation while the decay $\eta \rightarrow \pi^{+} \pi^{-} e^{+} e^{-}$offers the possibility to search for a possible flavor conserving $C P$ violation. The very rare decays of pseudoscalar mesons in a single lepton pair $\pi^{0}, \eta \rightarrow e^{+} e^{-}$finally allow to search for physics beyond the Standard Model.

In the following, the WASA facility at COSY will be introduced and selected analysis examples from the three fields of interest mentioned above will be discussed, to show, what can be learned form light meson decays.

\section{The WASA facility at COSY}

The WASA detection system [1] is a large acceptance spectrometer for charged and neutral particle detection. It is installed at an internal target position of the COoler SYnchrotron COSY [2] at the Forschungszentrum Jülich in Germany. COSY provides proton and deuteron beams with momenta up to $3.7 \mathrm{GeV} / \mathrm{c}$. The beams can be phase space cooled by means of electron cooling at lower and stochastic cooling at higher energies. Polarized beams are possible.

The WASA facility makes use of a pellet target system. Frozen droplets of liquid hydrogen or deuterium with a diameter of $35 \mu \mathrm{m}$ are used as target material and provide an effective target thickness of $10^{15}$ atoms $/ \mathrm{cm}^{2}$. In the interaction with the beams, luminosities in the order of $10^{31}-10^{32} \mathrm{~cm}^{-2} \mathrm{~s}^{-1}$ are achieved.

The detector is divided into a forward part covering scattering angles from $3-18$ degrees and a central detector covering the angular range from $20-160$ degrees. A cross section of the setup is shown in Fig. 1.

The central detector surrounds the interaction point. It is designed to detect the produced mesons and their decay particles. A cylindrical straw tube tracker is operated in the field of a superconducting solenoid to allow for momentum measurement of charged particles. It is surrounded 


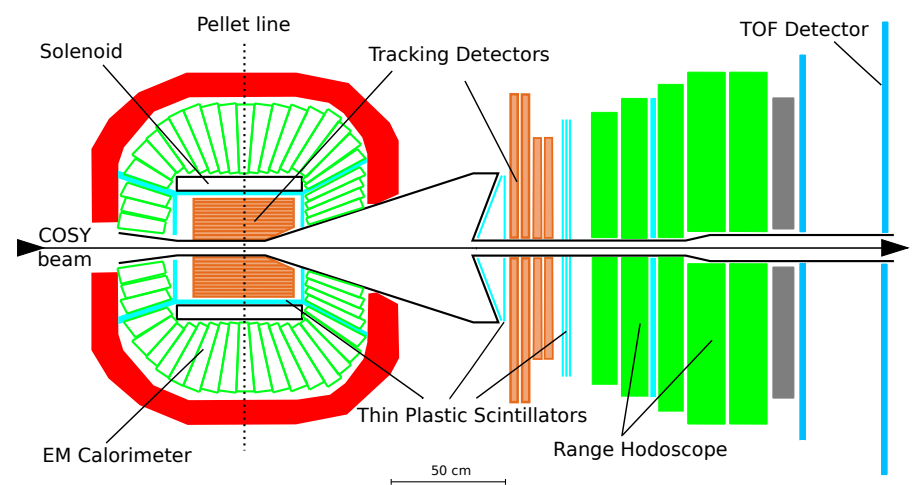

Figure 1: A cross section of the WASA detector setup. Central part of the spectrometer is on the left and the forward part on the right. COSY beam direction and pellet target position are indicated.

by an electromagnetic calorimeter made from $1012 \mathrm{CsI}(\mathrm{Na})$ crystals to measure energies of charged as well as neutral particles. A barrel of thin plastic scintillators is used to determine the timing of charged particles and to veto them on the trigger level.

The forward detectors are designed to measure the charged recoil particles from the meson production and to tag the produced mesons by their missing mass. The energy loss patterns in the thin and thick scintillators are used to identify particles, while the kinetic energies are reconstructed from the energy loss in the thick scintillators of the range hodoscope. Scattering angles are measured by a straw tube tracker.

Mesons are produced in proton-proton and proton-deuteron collisions. In the latter case the focus is on the fusion reaction $\mathrm{pd} \rightarrow{ }^{3} \mathrm{He} \mathrm{X}$, with $\mathrm{X}$ being the produced meson. Both production reactions are complementary to the performed decay studies. The higher cross section in the $\mathrm{pp} \rightarrow \mathrm{pp} \mathrm{X}$ reactions is favorable for studies of rare decays, but the generally large reaction rate requires selective triggers, which put a bias on the studied decay systems. The disadvantage of a lower production rate in proton-deuteron production is outweighed by the better acceptance due to a lower center of mass velocity, a smaller number of directly produced pions relative to the number of produced $\eta$ and $\omega$ mesons and the possibility to identify the ${ }^{3} \mathrm{He}$ ions by their energy loss on the trigger level. Production runs focusing on $\pi^{0}$ mesons are performed only in proton-proton collisions. The center of mass energy is chosen below the multi-pion threshold.

With the WASA facility at $\operatorname{COSY} 3 \cdot 10^{7} \eta$ mesons and $5 \cdot 10^{5} \omega$ mesons have been produced and tagged in the reaction pd $\rightarrow{ }^{3} \mathrm{He} \mathrm{X}$. In proton-proton collisions $3 \cdot 10^{8} \pi^{0}$ mesons have been tagged and more than $10^{8} \eta$ mesons have been produced.

\section{Meson Decay Studies}

\subsection{Precision Tests of ChPT}

The decay $\eta \rightarrow 3 \pi$ is an isospin violating process and allows experimental access to the light quark mass ratios. Additionally, a very important and precise tool to test the implementation of $\pi-\pi$ scattering in Chiral Perturbation Theory is provided by the comparison of the Dalitz plot densities to theory predictions. 
The amplitude of the three-pion decay can be described with the variables $X$ and $Y$, where

$$
X=\sqrt{3} \frac{T_{+}-T_{-}}{T_{+}+T_{-}+T_{0}} \quad Y=3 \frac{T_{0}}{T_{+}+T_{-}+T_{0}}-1,
$$

with $T_{+}, T_{-}, T_{0}$ being the kinetic energies of the charged and neutral pions in the $\eta$ rest frame. The Dalitz plot is parameterized by expanding the amplitude around $X, Y=0$ :

$$
|A(X, Y)|^{2} \propto 1+a Y+b Y^{2}+d X^{2}+f Y^{3}+g X^{2} Y+\ldots
$$

with $a, b, \ldots, g, \ldots$ being the Dalitz plot parameters.

In contrast to the decay $\eta \rightarrow 3 \pi^{0}$, where a strong database on the Dalitz plot parameters has been established over the recent years [4], only a single high statistics measurement is available for the decay $\eta \rightarrow \pi^{+} \pi^{-} \pi^{0}$ [5]. The Dalitz plot parameters $b$ and $f$, extracted by the KLOE collaboration based on $1.34 \cdot 10^{6}$ events, deviate significantly from Chiral Perturbation Theory calculations.

In order to bring the experimental database to similar accuracy and consistency as for the neutral three pion decay of the $\eta$ meson, two independent and competitive measurements are realized by the WASA-at-COSY collaboration. They are performed on the acquired proton-deuteron and proton-proton data, respectively.

Currently, $30 \%$ of the proton-deuteron data have been analyzed. Background subtracted and acceptance corrected Dalitz plot distributions have been extracted based on $2.1 \cdot 10^{5}$ reconstructed $\eta \rightarrow \pi^{+} \pi^{-} \pi^{0}$ events. A detailed report on the analysis can be found in the contribution of P. Adlarson to these proceedings [6]. Fig. 2 shows the total projection of the Dalitz plot on the $X$ and $Y$ axes. Along with the data the distributions are shown, which are expected from LO calculations (solid line) and NNLO calculations (dot-dashed line) of Chiral Perturbation Theory and the distribution according to the parameters extracted by the KLOE collaboration (dashed line). At the still preliminary stage, the data are more consistent with the KLOE measurement than with the theory predictions.
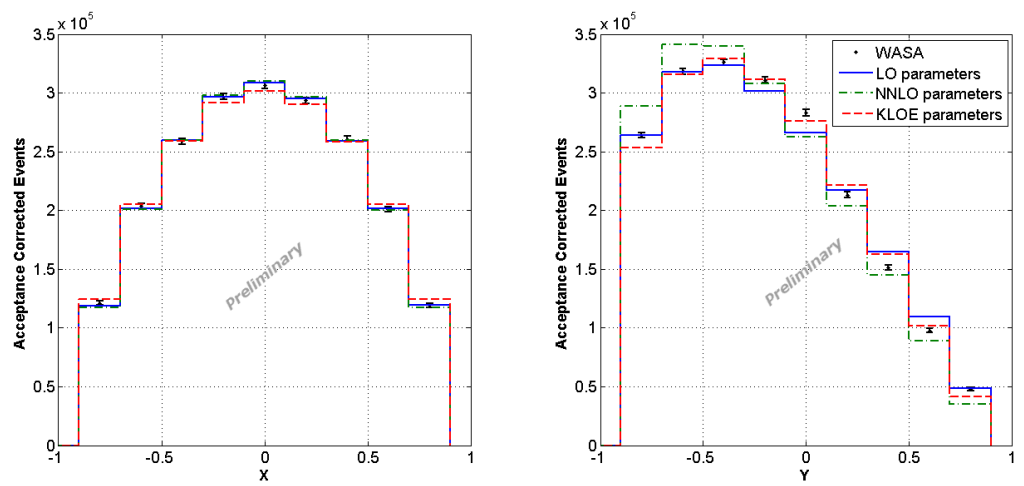

Figure 2: The background subtracted and acceptance corrected projections of the Dalitz plot of $\eta \rightarrow$ $\pi^{+} \pi^{-} \pi^{0}$. The data points are shown together with the distributions according to leading order (solid line), NNLO (dot-dashed line) calculations and according to the parameters of Ref. [5] (dashed line).

The anomalous sector of QCD can be addressed with the decay $\eta \rightarrow \pi^{+} \pi^{-} \gamma$. At the chiral limit the decay is completely described by the box anomaly term of the Wess-Zumino-Witten Lagrangian [7]. However, the phase space of the decay is confined by the rest masses of the $\eta$ meson 
and the charged pions. Thus, the decay is well above the chiral limit which is reflected in the significant deviation of the predicted decay rate from the experimental value. Various attempts have been made to include final-state interactions as unitarized extensions to the box anomaly term $[8,9]$. The predictions for both, the decay rate and the dynamics of the final state part, can be test, but so far experimental data especially on the dynamics were scarce [10] and yielded ambiguous interpretation [9].

Using proton-deuteron data corresponding to $10^{7}$ tagged $\eta$ mesons, background subtracted and acceptance corrected differential distributions have been extracted with the WASA detector [11]. The number of $13960 \pm 140$ reconstructed $\eta \rightarrow \pi^{+} \pi^{-} \gamma$ events comprises the largest exclusive sample used for the studies of the decay dynamics so far. The photon energy distribution, shown in Fig. 3 was found to be at variance with the simplest gauge invariant matrix element (dashed line) given by the box term. To describe the spectral shape a model independent ansatz has been used, following Ref. [12]. It allows to describe the distribution with a single free parameter $\alpha$. A fit to the data results in $\alpha=1.89 \pm 0.25_{\text {stat }} \pm 0.59_{\text {sys }} \pm 0.02_{\text {theo }} \mathrm{GeV}^{-2}$.

Recently, the CLEO collaboration extracted a relative branching ratio of $\eta \rightarrow \pi^{+} \pi^{-} \gamma$ [13], which differs by more than three standard deviations from the previous measurements [3]. A new measurement of the decay is currently performed at WASA, based on the data acquired in protonproton interactions. It will not only allow to determine the decay dynamics with much higher precision, but also the branching ratio.

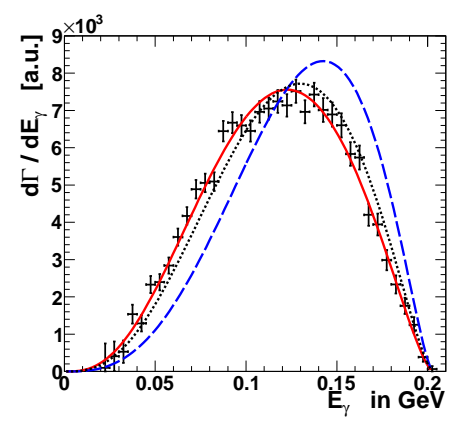

Figure 3: The background subtracted and acceptance corrected photon energy distribution of the decay $\eta \rightarrow \pi^{+} \pi^{-} \gamma$ [11]. The data points are fitted with the model independent form factor of Ref. [12] (solid line). For comparison the distributions predicted with a parameter $\alpha=0$ (dotted line) and predicted by the simplest matrix element (dashed line) are shown.

\subsection{Transition Form Factors}

The Dalitz decays $\pi^{0}, \eta \rightarrow e^{+} e^{-} \gamma$ proceed via the internal conversion of a time-like virtual photon into a lepton pair. The transition form factors of the light mesons describe the difference of the decay rate relative to the solution for point-like particles given by QED. Thus, the form factor reflects the spatial electromagnetic structure arising at the decay of the neutral mesons. It is also an important input to the Standard Model contributions in the calculation of $g-2$ of the muon.

A detailed report on the analysis of the Dalitz decay $\pi^{0} \rightarrow e^{+} e^{-} \gamma$ can be found in the contribution of C.-O. Gullström to these Proceedings [14]. The transition form factor of the $\eta$ meson has recently been measured with high statistics by the NA60 collaboration [15] in the decay 
$\eta \rightarrow \mu^{+} \mu^{-} \gamma$. However, the final state particles have not been measured exclusively. Instead, the inclusive dimuon mass spectrum measured by the heavy ion experiment has been unfolded using a number of models.

At WASA, $\eta$ mesons are produced in elementary reactions and the decay is measured exclusively. Main sources of background to the invariant mass spectrum of the lepton pairs stems from photon conversion in the detector material in the lower mass region and from misidentified pions in the higher mass region. Background from external conversion can be effectively suppressed by conditions on the vertex position. Contributions from misidentified pions are reduced by imposing energy and momentum conservation and by the subtraction of prompt multi-pion background, which is not stemming from $\eta$ decays. A number of 650 and $3000 \eta \rightarrow e^{+} e^{-} \gamma$ events has been extracted from $30 \%$ of the proton-deuteron and $20 \%$ of the acquired proton-proton data, respectively. In the next steps the acceptance correction will be performed and the influence of possible systematic effects will be studied.

The double Dalitz decay $\eta \rightarrow e^{+} e^{-} e^{+} e^{-}$allows to extract the transition form factor $F\left(q_{1}, q_{2}\right)$ based on two virtual photons. As a fourth-order electromagnetic process, the decay has a branching ratio as low as $2.6 \cdot 10^{-5}$, as predicted by QED calculations. Recently, for the first time the branching ratio was measured and found to be in agreement with the QED prediction [16].

Two independent analyses have been performed on the proton-deuteron data measured with the WASA detector. Different emphasis has been put on the decay dynamics as well as the topology and the unambiguous identification of the final state particles, respectively. Both analyses report consistently $50 \pm 14$ event candidates and extracted a preliminary branching ratio of $\left(3.0 \pm 0.8_{\text {stat }} \pm 0.7_{\text {sys. (norm) }}\right) \cdot 10^{-5}$. This value is in agreement with both, the theoretical and experimental values. Currently systematic studies of the individual results as well as consistency checks between both analyses are being performed. The final goal is to probe the transition form factor $F\left(q_{1}, q_{2}\right)$ in the analysis of all data acquired with the WASA detector at COSY.

\subsection{Tests of Standard Model Predictions}

The decays of $\pi^{0}$ and $\eta$ into a single dilepton pair are described in the Standard Model by a two-photon process. Relative to the two-photon decay the branching ratios are suppressed by $\alpha^{2}$ and the helicity conservation, which contributes with a factor $\left(\frac{m_{e}}{m_{\pi, \eta}}\right)^{2}$. The resulting branching ratios are $(6.2 \pm 0.1) \cdot 10^{-8}$ and $(4.6 \pm 0.1) \cdot 10^{-9}$, respectively [17]. A significant deviation from these values might hint at physics beyond the Standard Model.

In case of the decay $\pi^{0} \rightarrow e^{+} e^{-}$a branching ratio of $(7.49 \pm 0.29) \cdot 10^{-8}$ has been measured by the KTeV Collaboration [18]. The excess of three standard deviations compared to the Standard Model prediction leads to some speculation about new physics. A promising theory, which would also explain phenomena observed in cosmology, is the existence of a new gauge boson $U$ that mediates the annihilation of light dark matter into lepton pairs [19]. A coupling of $U$ to quarks and leptons could also account for the excess observed for the $\pi^{0} \rightarrow e^{+} e^{-}$decay.

In spring 2010 a precursor experiment has been performed with the WASA facility at COSY. From the data taken during one week 15 event candidates of the $\pi^{0} \rightarrow e^{+} e^{-}$decay could be identified after background subtraction. The main source of background is the Dalitz decay $\pi^{0} \rightarrow \gamma e^{+} e^{-}$, where the photon remains undetected. Details of the analysis are presented in the contribution of 
C.-O. Gullström to these proceedings [14]. The positive result of the precursor studies motivate longer production runs. Tests showed that with improved experimental conditions even 50 event candidates can be measured per week.

For the branching ratio of the decay $\eta \rightarrow e^{+} e^{-}$only an experimental upper limit is known. The best value was established by the CELSIUS/WASA collaboration to be $7.7 \cdot 10^{-5}$ at $90 \%$ CL [3]. In the analysis of data taken in two weeks of proton-proton interactions with the WASA facility at COSY the upper limit could already be improved by one order of magnitude. The analyzed amount of data corresponds approximately to $20 \%$ of all acquired data on $\eta$ decays. An improvement by yet another order of magnitude is expected from the analysis of the complete data set. The goal for future $\eta$ production runs is to achieve standard model sensitivity.

\section{Acknowledgments}

This publication has been supported by the European Commission under the 7th Framework Programme through the 'Research Infrastructures' action of the 'Capacities' Programme. Call: FP7-INFRASTRUCTURES-2008-1, Grant Agreement N. 227431 and by the Polish National Science Centre and Foundation for Polish Science - MPD program, co-financed by the European Union within the European Regional Development Fund.

\section{References}

[1] H.-H. Adam et al. [WASA-at-COSY Coll.], arXiv: nucl-ex/0411038.

[2] R. Maier Nucl. Instrum. Meth. A390 (1997) 1-8.

[3] K.Nakamura et.al. (Particle Data Group), J. of Phys. G37, 0705021 (2010) and 2011 partial update for the 2012 edition.

[4] A. Abele et al. [Crystal Barrel Coll.], Phys. Lett., B 417, 193, (1998).

F. Ambrosino et al. [KLOE Coll.], Phys.Lett., B 694, 16, (2010).

Crystal Ball Collaboration, Phys. Rev. Lett., 87, 192001, (2001).

M. Bashkanov et al. [CELSIUS/WASA Coll.], Phys. Rev., C 76, 048201, (2007).

C. Adolph et al. [WASA-at-COSY Coll.], Phys. Lett., B 677, 24, (2009).

Crystal Ball at MAMI Coll., Eur. Phys. J., A 39, 169, (2009).

Crystal Ball at MAMI Coll., Phys. Rev., C 79, 035204, (2009).

[5] F. Ambrosino et al. [KLOE Coll.], JHEP 0805 (2008) 006.

[6] P. Adlarson, contribution to these proceedings.

[7] J. Wess, B. Zumino, Phys. Lett. B 37, 95 (1975).

E. Witten, Nucl. Phys. B 223, 422 (1983).

[8] J. Bijnens, A. Bramon, and F. Cornet Phys. Lett. B237 (1990) 488.

C. Picciotto Phys. Rev. D45 (1992) 1569-1574.

E. P. Venugopal and B. R. Holstein Phys. Rev. D57 (1998) 4397-4402.

B. R. Holstein Phys. Scripta T99 (2002) 55-67.

[9] M. Benayoun, et al. Eur. Phys. J. C31 (2003) 525-547.

B. Borasoy and R. Nißler Nucl. Phys. A740 (2004) 362-382. 
[10] F. S. Crawford and L. R. Price Phys. Rev. Lett. 16 (1966) 333.

A. M. Cnops et al. Phys. Lett. 26B (1968) 398.

M. Gormley et al. Phys. Rev. D2 (1970) 501-505.

J. G. Layter et al. Phys. Rev. D7 (1973) 2565-2568.

[11] P. Adlarson et al. [WASA-at-COSY Coll.], Phys. Lett. B, 707243 (2012).

[12] F. Stollenwerk, C. Hanhart, A. Kupsc, U.-G. Meißner, A. Wirzba, Phys. Lett. B, 707184 (2012).

[13] A. Lopez et al. [CLEO Coll.] Phys. Rev. Lett. 99 (2007) 122001,

[14] C.-O. Gullström, contribution to these proceedings

[15] R. Arnaldi et al. [NA60 Coll.], Phys. Lett. B, 677, 260 (2009).

[16] F. Ambrosino et al. [KLOE Coll.], Phys. Let. B, 702, 324 (2011).

[17] A. Dorokhov, M. A. Ivanov, Phys. Rev. D 75 (2007) 114007.

[18] E. Abouzaid et. al, Phys. Rev. D 75 (2007) 012004.

[19] C. Boehm and P.Fayet, Nucl. Phys. B 683 (2004) 219. Y Kahn, M. Schmitt, T.M.P. Tait, Phys. Rev. D 78 (2008) 115002. 